\title{
ON THE YANG-BAXTER EQUATION AND LEFT NILPOTENT LEFT BRACES
}

\author{
FERRAN CEDÓ, TATIANA GATEVA-IVANOVA AND AGATA SMOKTUNOWICZ
}

\begin{abstract}
We study non-degenerate involutive set-theoretic solutions $(X, r)$ of the Yang-Baxter equation, we call them simply solutions. We show that the structure group $G(X, r)$ of a finite non-trivial solution $(X, r)$ cannot be an Engel group. It is known that the structure group $G(X, r)$ of a finite multipermutation solution $(X, r)$ is a poly- $\mathbb{Z}$ group, thus our result gives a rich source of examples of braided groups and left braces $G(X, r)$ which are poly- $\mathbb{Z}$ groups but not Engel groups.

We also show that a finite solution of the Yang-Baxter equation can be embedded in a convenient way into a finite brace and into a finite braided group.

For a left brace $A$, we explore the close relation between the multipermutation level of the solution associated with it and the radical chain $A^{(n+1)}=$ $A^{(n)} * A$ introduced by Rump.
\end{abstract}

\section{INTRODUCTION}

Braces were introduced by Rump [15] to study non-degenerate involutive settheoretic solutions of the Yang-Baxter equation.

Recall that a left brace is a set $B$ with two operations, + and $\cdot$, such that $(B,+)$ is an abelian group $(B, \cdot)$ is a group and for every $a, b, c \in B$,

$$
a \cdot(b+c)+a=a \cdot b+a \cdot c .
$$

Right braces are defined similarly, changing the property (11) by $(a+b) \cdot c+c=$ $a \cdot c+b \cdot c$. A two-sided brace is a left brace which is also a right brace. In any left brace $(B,+, \cdot)$ one defines another operation $*$ by the rule

$$
a * b=a \cdot b-a-b,
$$

for $a, b \in B$. It is known that $(B,+, \cdot)$ is a two-sided brace if and only if $(B,+, *)$ is a Jacobson radical ring. Conversely, if $R$ is a Jacobson radical ring, then one

Date: January 27, 2016.

2010 Mathematics Subject Classification. Primary 16N80, 16P90, 16N40 .

Key words and phrases. brace, Yang-Baxter equation, nilpotent braces. 
defines a new operation $\circ$ on $R$ by $a \circ b=a b+a+b$ and $(R, \circ)$ is called the adjoint group of the radical ring $R$. Then $(R,+, \circ)$ is a two-sided brace. Hence the study of two-sided braces is equivalent to the study of Jacobson radical rings.

In general the operation $*$ in a left brace $B$ is not associative, but it is left distributive with respect to the sum, that is

$$
a *(b+c)=a * b+a * c,
$$

for $a, b, c \in B$.

Let $B$ be a left brace. For $a \in B$, let $\mathcal{L}_{a}: B \longrightarrow B$ be the map defined by $\mathcal{L}_{a}(b)=a b-a$ for all $b \in B$. It is known that $\mathcal{L}_{a}$ is an automorphism of the additive group of the left brace $B$ and the map $\mathcal{L}:(B, \cdot) \longrightarrow \operatorname{Aut}(B,+)$, defined by $a \mapsto \mathcal{L}_{a}$, is a morphism of groups. The kernel of this morphism is called the socle of $B$,

$$
\operatorname{Soc}(B):=\left\{a \in B \mid \mathcal{L}_{a}=\mathrm{id}\right\}=\{a \in B \mid a b=a+b, \text { for all } b \in B\} .
$$

In fact the socle of a left brace $B$ is an ideal of $B$, that is, a normal subgroup of its multiplicative group invariant by the maps $\mathcal{L}_{a}$ for all $a \in B$. In particular, $\operatorname{Soc}(B)$ is also a subgroup of the additive group of $B$. Note that if $a, b \in \operatorname{Soc}(B)$, then $a-b=\mathcal{L}_{b}\left(b^{-1} a\right) \in \operatorname{Soc}(B)$. Therefore the quotient of the multiplicative group $B / \operatorname{Soc}(B)$ is also the quotient of the additive group and $(B / \operatorname{Soc}(B),+, \cdot)$ is a left brace, the left brace quotient of $B$ modulo its ideal $\operatorname{Soc}(B)$.

Let $X$ be a non-empty set. Recall that a map $r: X \times X \longrightarrow X \times X$ is a set-theoretic solution of the Yang-Baxter equation if

$$
r_{12} r_{23} r_{12}=r_{23} r_{12} r_{23},
$$

where $r_{12}, r_{23}: X \times X \times X \longrightarrow X \times X \times X$ are the maps $r_{12}=r \times \operatorname{id}_{X}$ and $r_{23}=\mathrm{id}_{X} \times r$. We will write $r(x, y)=\left({ }^{x} y, x^{y}\right)$. The map $r$ is non-degenerate if for every $x \in X$ the maps $y \mapsto{ }^{x} y$ and $y \mapsto y^{x}$ are bijective, $r$ is involutive if $r^{2}=\operatorname{id}_{X^{2}}$.

Convention. By a solution of the YBE (or shortly, a solution) we mean a nondegenerate involutive set-theoretic solution $(X, r)$ of the Yang-Baxter equation.

Let $(X, r)$ be a solution of the YBE. The structure group of $(X, r)$ is the group $G(X, r)$ with presentation

$$
G(X, r)=\left\langle X \mid x y=\left({ }^{x} y\right)\left(x^{y}\right), x, y \in X\right\rangle .
$$

(Some authors call $G(X, r)$ the YB group of $(X, r)$ ). 
It follows from the results in 8 that $X$ is naturally embedded in $G(X, r)$. One can define a sum on $G(X, r)$ such that $(G(X, r),+)$ is a free abelian group with basis $X$. Moreover, $(G(X, r),+, \cdot)$ is a left brace such that ${ }^{x} y=\mathcal{L}_{x}(y) \in X$ for all $x, y \in X$, see [5, 9].

We say that this is the canonical left brace structure on $G(X, r)$. It is known that the group $G$ acts on the set $X$ from the left (and from the right). Moreover, the assignments $x \mapsto \mathcal{L}_{x}$ extend to a group homomorphism $\mathcal{L}: G \longrightarrow \operatorname{Sym}_{X}$. The image $\mathcal{L}(G)$ of this homomorphism is a subgroup of $\operatorname{Sym}_{X}$ called the permutation group of $(X, r)$ and denoted by $\mathcal{G}(X, r)$. It is known that $\mathcal{G}(X, r):=$ $\left\langle\mathcal{L}_{x} \mid x \in X\right\rangle$, where $\mathcal{L}_{x}(y)={ }^{x} y$, for all $x, y \in X$. The group epimorphism $\mathcal{L}: G(X, r) \longrightarrow \mathcal{G}(X, r), x \mapsto \mathcal{L}_{x}$ has kernel $\operatorname{Ker} \mathcal{L}=\operatorname{Soc}(G(X, r))$ (as sets). Thus $\mathcal{G}(X, r)$ inherits a structure of a left brace via this natural isomorphism of groups, we say that this is the canonical structure of a left brace on $\mathcal{G}(X, r)$. Moreover, $G(X, r) / \operatorname{Soc}(G(X, r)) \cong \mathcal{G}(X, r)$ as symmetric groups (i.e. involutive braided groups) and as left braces, [9, 5].

In this paper, we prove some general results about braces and apply these to study the close relations between the properties of solutions $(X, r)$ and their associated left braces $G(X, r)$. This is in the spirit of [9] and [5].

\section{Some Results on $G(X, r)$}

The results of this section were motivated by a result from [9, which assures that the structure group $G(X, r)$ of a non-trivial solution $(X, r)$ of the Yang Baxter cannot be a two-sided brace.

Let $(B,+, \cdot)$ be a left brace. As usual, for any $a, b \in B$ and positive integer $m$, $a b$ will denote $a \cdot b$ and $a^{m}$ will denote $a \cdot a \cdots a$ (where $a$ appears $m$ times).

Lemma 1. Let $B$ be a left brace whose additive group $(B,+)$ is torsion-free. Assume that $a, b \in B$ and that there is an integer $n(a, b)$ such that $a *(a *(\ldots a *$ $(a * b) \ldots))=0$ (where a occurs $n(a, b)$ times and $b$ once in this equation). Assume moreover that $\mathcal{L}_{a^{n}}=i d$ for some integer $n$. Then $a * b=0$ or equivalently, $a \cdot b=a+b$.

Proof. Note that $\mathcal{L}_{a}(b)=a * b+b$, for $a, b \in B$. Let $m$ be a positive integer. Let $e_{1}(a, b)=a * b$ and $e_{m+1}(a, b)=a * e_{m}(a, b)$, for $a, b \in B$. It can be proved by induction on $m$ that

$$
\mathcal{L}_{a^{m}}(b)=b+\sum_{i=1}^{m}\left(\begin{array}{c}
m \\
i
\end{array}\right) e_{i}(a, b) .
$$


Since $\mathcal{L}_{a^{n}}=$ id, we have

$$
b=\mathcal{L}_{a^{n}}(b)=b+\sum_{i=1}^{n}\left(\begin{array}{c}
n \\
i
\end{array}\right) e_{i}(a, b)
$$

and thus

$$
n e_{1}(a, b)=-\sum_{i=2}^{n}\left(\begin{array}{c}
n \\
i
\end{array}\right) e_{i}(a, b)
$$

Hence

$$
n e_{k}(a, b)=-\sum_{i=2}^{n}\left(\begin{array}{c}
n \\
i
\end{array}\right) e_{i+k-1}(a, b)
$$

for all positive integer $k$.

Suppose that $e_{1}(a, b)=a * b \neq 0$. Let $n(a, b)$ be the smallest positive integer such that $e_{n(a, b)}(a, b)=0$. Then, by (2),

$$
n e_{n(a, b)-1}(a, b)=-\sum_{i=2}^{n}\left(\begin{array}{l}
n \\
i
\end{array}\right) e_{i+n(a, b)-2}(a, b)=0 .
$$

Since $(B,+)$ is torsion-free, we have that $e_{n(a, b)-1}(a, b)=0$, in contradiction with the definition of $n(a, b)$.

Let $G$ be a group. Following the notation of [14, for $g, h \in G$, we denote by $[g, h]$ the element $[g, h]=g^{-1} h^{-1} g h$. Recall that the group $G$ is an Engel group if and only if for each $g, h \in G$ there exists a positive integer $n(g, h)$ such that $[[\ldots[[g, h], h] \ldots], h]=1$, where $h$ occurs $n(g, h)$ times.

Theorem 2. Let $B$ be a left brace such that its additive group $(B,+)$ is torsionfree and $[B: \operatorname{Soc}(B)]=n<\infty$. If the multiplicative group $(B, \cdot)$ of the left brace $B$ is an Engel group, then $B$ is a trivial brace, that is $a \cdot b=a+b$, for all $a, b \in B$.

Proof. Let $a \in B$ and $c \in \operatorname{Soc}(B)$. Note that

$$
\mathcal{L}_{a}(c)=a c-a=a c a^{-1} a-a=a c a^{-1}+a-a=a c a^{-1} .
$$

Hence

$$
\begin{aligned}
{[a, c] } & =a^{-1} c^{-1} a c=a^{-1} c^{-1} a+c \\
& =\mathcal{L}_{a^{-1}}\left(c^{-1}\right)+c=\mathcal{L}_{a^{-1}}(-c)+c \\
& =-\mathcal{L}_{a^{-1}}(c)+c=-a^{-1} * c-c+c \\
& =-a^{-1} * c=\left(a^{-1} * c\right)^{-1} .
\end{aligned}
$$


Hence $[c, a]=a^{-1} * c$. Therefore $[[\ldots[[c, a], a] \ldots], a]=a^{-1} *\left(\ldots a^{-1} *\left(a^{-1} * c\right) \ldots\right)$. Let $b \in B$. Since $[B: \operatorname{Soc}(B)]=n<\infty$ and $\operatorname{Soc}(B)$ is an ideal of $B, n b \in \operatorname{Soc}(B)$. Since $(B, \cdot)$ is an Engel group, there exists a positive integer $m$ such that

$$
[[\ldots[[(n b), a], a] \ldots], a]=1,
$$

(where $a$ occurs $m$ times). Hence

$$
0=1=a^{-1} *\left(\ldots a^{-1} *\left(a^{-1} *(n b)\right) \ldots\right)=n\left(a^{-1} *\left(\ldots a^{-1} *\left(a^{-1} * b\right) \ldots\right)\right),
$$

(where $a^{-1}$ appears $m$ times). But $(B,+)$ is torsion free, hence

$$
a^{-1} *\left(\ldots a^{-1} *\left(a^{-1} * b\right) \ldots\right)=0,
$$

where $a^{-1}$ occurs $m$ times.

By Lemma 1, $a^{-1} * b=0$. Therefore $a * b=0$, for all $a, b \in B$, or equivalently, $B$ is a trivial left brace.

We call a left brace $B$ left nilpotent if $B^{n}=0$ for some $n$, where $B^{n+1}=B * B^{n}$ is the chain introduced by Rump in [15]. As a consequence of Lemma 1 and Theorem 2, we have the following two results.

Theorem 3. Let $(X, r)$ be a finite solution of the YBE. Assume that for each $a, b \in X$ there is a positive integer $n=n(a, b)$ such that the equality $a *(a *(\ldots a *$ $(a * b)))=0$ holds in $G(X, r)$,(a occurs $n$ times and $b$ occurs once in this equality). Then $(X, r)$ is the trivial solution. In particular, if $G(X, r)$ is a left nilpotent left brace, then $(X, r)$ is the trivial solution.

Proof. Since $G(X, r) / \operatorname{Soc}(G(X, r)) \cong \mathcal{G}(X, r)$ is a subgroup of the symmetric group $\operatorname{Sym}_{X}$ of the finite set $X$, we have that $[G(X, r): \operatorname{Soc}(G(X, r))]<\infty$. Hence, by Lemma 1 ${ }^{a} b=\mathcal{L}_{a}(b)=a b-a=a+b-a=b$, for all $a, b \in X$. In particular, $(X, r)$ is the trivial solution.

It is known that any ordered abelian-by-finite group is abelian, see for example [12, Section 4]. It is also known that any torsion-free nilpotent group is ordered (see [13, Lemma 13.1.6]). Recall that if $(X, r)$ is a finite solution of the YBE, then $G(X, r)$ is a torsion-free, solvable and abelian-by-finite group (see [8] and [10]). Therefore, if $G(X, r)$ is nilpotent, then it is abelian. In this case the canonical left brace structure on $G(X, r)$ is trivial and $(X, r)$ is the trivial solution. We have the following related result.

Theorem 4. Let $(X, r)$ be a finite solution of the YBE. If the structure group $G(X, r)$ is an Engel group, then $(X, r)$ is the trivial solution. 
Proof. This is a consequence of Theorem 2

\section{Right nilpotent Left BRACES}

Etingof, Schedler and Soloviev in [8] introduced the retract solution of a given solution of the YBE. Let $(X, r)$ be a solution of the YBE. The retract relation $\sim$ on the set $X$ with respect to $r$ is defined by $x \sim y$ if $\sigma_{x}=\sigma_{y}$, where $\sigma_{x}(z)={ }^{x} z$. Then the retraction of $(X, r)$ is $\operatorname{Ret}(X, r)=\left([X], r_{[X]}\right)$, where $[X]=X / \sim$ and

$$
r_{[X]}([x],[y])=\left(\left[{ }^{x} y\right],\left[x^{y}\right]\right),
$$

where $[x]$ denotes the $\sim$-class of $x \in X$. We define $\operatorname{Ret}^{1}(X, r)=\operatorname{Ret}(X, r)$ and $\operatorname{Ret}^{k}(X, r)=\operatorname{Ret}\left(\operatorname{Ret}^{k-1}(X, r)\right)$ for $k>1$. A solution $(X, r)$ of the YBE is called a multipermutation solution of level $m$ if $m$ is the smallest nonnegative integer such that the solution $\operatorname{Ret}^{m}(X, r)$ has cardinality 1 ; in this case we write $\operatorname{mpl}(X, r)=m$.

Let $B$ be a left brace. By $B^{(m)}$ we mean the chain of ideals introduced by Rump in [15], so $B^{(1)}=B$ and $B^{(n+1)}=B^{(n)} * B$. We say that $B$ is right nilpotent if there exists a positive integer $n$ such that $B^{(n)}=0$.

Recall that if $B$ is a left brace, then the map $r: B \times B \longrightarrow B \times B$ defined by

$$
r(a, b)=\left(\mathcal{L}_{a}(b), \mathcal{L}_{\mathcal{L}_{a}(b)}^{-1}(a)\right),
$$

is a solution of the YBE. This is the solution of the YBE associated with the left brace $B$ (see [6]).

Proposition 5. Let $B$ be a nonzero left brace and let $(B, r)$ be its associated solution of the YBE. Then the multipermutation level of $(B, r)=m<\infty$ if and only if $B^{(m+1)}=0$ and $B^{(m)} \neq 0$.

Proof. Note that $\operatorname{Soc}(B)=\{b \in B \mid b * a=0$ for every $a \in B\}$.

First we shall prove the implication $(\operatorname{mpl}(B, r)=m) \Rightarrow\left(B^{(m+1)}=0\right.$ and $\left.B^{(m)} \neq 0\right)$. We use induction on $m=\operatorname{mpl}(B, r)$. Suppose $\operatorname{mpl}(B, r)=1$. Therefore, $\mathcal{L}_{a}(b)=a * b+b=b$ which is equivalent to $a * b=0$ for all $a, b \in B$. It follows that $B * B=0$, so $B^{(2)}=0$. But $B$ is a nonzero left brace, hence $B^{(1)}=B \neq 0$. This gives the base for induction.

Suppose now that for all $k, 1 \leq k \leq m-1$, the condition $\operatorname{mpl}(B, r)=k \leq m-1$ implies $B^{(k+1)}=0$ and $B^{(k)} \neq 0$. Assume that $\operatorname{mpl}(B, r)=m$, then the retraction $\operatorname{Ret}(B, r)=\left([B], r_{[B]}\right)$ has multipermutation level $m-1$.

Moreover, there is an isomorphism of left braces (or equivalently an isomorphism of braided groups) $B / \operatorname{Soc}(B) \cong[B]$ and $\operatorname{Ret}(B, r)$ is isomorphic to the solution of the YBE associated with $B / \operatorname{Soc}(B)$ ([15], [6], 9]). Hence by the inductive 
assumption $(B / \operatorname{Soc}(B))^{(m)}=0$ and $(B / \operatorname{Soc}(B))^{(m-1)} \neq 0$. This implies $B^{(m)} \subseteq$ $\operatorname{Soc}(B)$ and that $B^{(m-1)}$ is not a subset of $\operatorname{Soc}(B)$. Therefore $B^{(m+1)}=0$ and $B^{(m)} \neq 0$.

Now we prove the inverse implication: $\left(B^{(m+1)}=0\right.$ and $\left.B^{(m)} \neq 0\right) \Rightarrow(\operatorname{mpl}(B, r)=$ $m)$.

The base for the induction is clear. Assume that for all $k \leq m$ the implication is true. Suppose that $B$ is a left brace such that $B^{(m+2)}=0$ and $B^{(m+1)} \neq 0$. Recall that $B^{(m+2)}=B^{(m+1)} * B$, therefore $(B / \operatorname{Soc}(B))^{(m+1)}=0$. On the other hand $B^{(m+1)} \neq 0$ and $B^{(m+1)}=B^{(m)} * B$ imply $(B / \operatorname{Soc}(B))^{(m)} \neq 0$. By the inductive assumption $\operatorname{mpl}(\operatorname{Ret}(B, r))=m$, and therefore, $\operatorname{mpl}(B, r)=m+1$. This proves the proposition.

\section{Embedding solutions AND GROUps into Finite BRACES AND FINITE RINGS}

In this section we will show that a finite solution of the YBE can be embedded (in an explicit way) into a finite left brace. Recall that it was shown in 9 that there is a canonical one-to-one correspondence between left braces and symmetric groups (in the sense of Takeuchi [17]). Therefore Proposition[6]also shows explicitly how to embed a finite solution of the YBE into a finite symmetric group.

Proposition 6. Let $(X, r)$ be a finite solution of the $Y B E$. Then there is a finite left brace $(B,+, \cdot)$ such that $X \subseteq B$ generates the additive group of $B$. Moreover, $(X, r)$ is a subsolution of the solution $(B, \sigma)$ associated canonically with the left brace $B$, that is, $X$ is $\sigma$-invariant and $r=\sigma_{\mid X \times X}$ is the restriction of $\sigma$ on $X \times X$.

Proof. Let $G=G(X, r)$ be the structure group of the finite solution $(X, r)$. We know that the additive group of the left brace $(G,+,$.$) associated with G$ is free abelian with basis $X$ and ${ }^{a} b=\mathcal{L}_{a}(b)=a b-a$ and $a^{b}=\mathcal{L}_{\mathcal{L}_{a}(b)}^{-1}(a)$, for all $a, b \in X$. Since $X$ is finite, $[G: \operatorname{Soc}(G)]<\infty$, say $[G: \operatorname{Soc}(G)]=n$. Consider the set $I=\{n g \mid g \in G(X, r)\}$. We claim that $I$ is an ideal of the brace $G$, that is, $I$ is a normal subgroup of the multiplicative group $(G,$.$) which is invariant with respect$ to the left actions by elements of $G, 5$. It is clear that $I$ is an additive subgroup of $(G,+)$ and $I \subseteq \operatorname{Soc}(G(X, r))$. Then for $u, v \in I$ one has $u v=u+{ }^{u} v=u+v \in I$, $u^{-1}=-u \in I$, so $I$ is a subgroup of $G$. Let $g, h \in G$. Then

$$
h(n g) h^{-1}=\left({ }^{h}(n g)\right)\left(h^{n g}\right)\left(h^{-1}\right)=\left({ }^{h}(n g)\right)\left(h h^{-1}\right)={ }^{h}(n g)=n(h g) \in I .
$$

Thus, $I$ is a normal subgroup of $(G,$.$) which is also invariant under the left action$ by elements of $G$. Therefore $I$ is an ideal of the left brace $(G,+,$.$) . It is not difficult$ 
to show that brace quotient $B=G / I$ is a finite left brace of order $n^{m}$, where $m$ is the cardinality of $X$. Observe also that for any two elements $x, y \in X, x \neq y$, one has $x-y \notin I$, since the additive group of $(G,+)$ is free abelian with a basis $X$. Now the restriction of the natural map $G \rightarrow G / I=B$ on the set $X$ is injective. The proposition has been proved.

At a conference in Porto Cesareo, B. Amberg mentioned that he and his collaborators first became interested in Jacobson radical rings because they gave them a way to construct examples of triply-factorizable groups. Later, they found more ways of constructing such examples. Triply factorized groups can be also used to define braces; see [16, Theorem 18]. Interesting results on triply factorized groups can be found in [1, 2, 3, 4, 16, 11. Triply factorized groups are for example useful for investigating the structure of normal subgroups of a group $G=A B$ which is a product of two subgroups. Several authors investigated connections between triply factorized groups and nearrings [16], [11. It might be interesting to investigate the connections between nearrings and braces. We would like to pose a related open question:

Question 1. Investigate whether there is any relation between nearrings and solutions of the YBE?

The multiplicative group of a brace $A$ is also called an adjoint group of brace $A$. Observe that [7, Corollary 3.6] asserts that every finite solvable group is a subgroup of an adjoint group of some left brace. We also make the following simple remark which follows from [5, Lemma 8.1] and [7, Corollary 3.8].

Remark. (Related to [7, Corollary 3.8] and [6, Lemma 8.1]) Every finite nilpotent group is a subgroup of the adjoint group of a finite nilpotent ring.

Let $p$ be a prime. By [6, Lemma 8.1], every finite $p$-group is isomorphic to a subgroup of the adjoint group of a finite nilpotent ring $R$ such that $R$ has cardinality a power of $p$. Let $G$ be a finite nilpotent group. Let $p_{1}, \ldots p_{m}$ be the the distinct prime divisors of the order of $G$. Let $P_{i}$ be the Sylow $p_{i}$-subgroup of $G$. Then $P_{i}$ is isomorphic to a subgroup of the adjoint group of a finite nilpotent ring $R_{i}$. Since $G \cong P_{1} \times \cdots \times P_{m}$, it is clear that $G$ is isomorphic to a subgroup of the adjoint group of the finite nilpotent ring $R_{1} \times \cdots \times R_{m}$. If $R$ is a ring, then the adjoint semigroup of $R$ is defined by $a \circ b=a+b+a \cdot b$.

By following the technique of the proof of [6, Lemma 8.1] we get the following result. 
Proposition 7. Let $G$ be a group and let $R$ be a ring (with unit). Then $G$ is isomorphic to a subgroup of the adjoint semigroup of the group ring $R[G]$.

Proof. Let $f: G \longrightarrow R[G]$ be the map defined by $f(g)=g-1$, for $g \in G$. Clearly $f$ is injective. Let $g, h \in G$. We have that

$f(g h)=g h-1=(g-1)(h-1)+g-1+h-1=(g-1) \circ(h-1)=f(g) \circ f(h)$.

Therefore $f$ is an injective homomorphism of semigroups from $G$ into the adjoint semigroup of $R[G]$.

\section{ACKNOWLEDGMENTS}

The research of the first author was partially supported by grants DGI MICIIN MTM2011-28992-C02-01 and MINECO MTM2014-53644-P. The research of the second author was partially supported by Grant I 02/18 "Computational and Combinatorial Methods in Algebra and Applications" of the Bulgarian National Science Fund, by The Abdus Salam International Centre for Theoretical Physics (ICTP), Trieste, and by Max-Planck Institute for Mathematics, Bonn. The research of the third author was supported with ERC grant 320974.

\section{REFERENCES}

[1] B. Amberg, Triply factorized groups in the Groups St Andrews 1989, Vol 1, London Mathematical Society Lecture Note Series, Cambridge University Press 1991, 1-13.

[2] B.Amberg, S. Franciosi, F. de Giovanni, Triply factorized groups, Communications in Algebra Volume 18, Issue 3, 1990, 789-809.

[3] B. Amberg, L. Kazarin, Nilpotent p-algebras and factorized p-groups, Proceedings of "Groups St. Andrews 2005" , London Math. Society Lecture Note Series 339, Vol 1, Cambridge Univ. Press (2007), 130-147.

[4] B. Amberg, Ya.P. Sysak, Radical rings and products of groups, Groups St Andrews 1997 in Bath: Edited by C. M. Campbell, 1-19.

[5] Ferran Cedó, Eric Jespers, Jan Okniński, Braces and the Yang-Baxter Equation, arXiv:1205.3587 1 [math.RA].

[6] Ferran Cedó, Eric Jespers, Jan Okniński, Braces and the Yang-Baxter Equation, Communications in Mathematical Physics April 2014, Volume 327, Issue 1, pp 101-116.

[7] Ferran Cedó, Eric Jespers, Ángel de Río, Involutive Yang-Baxter groups, Trans. Amer. Math. Soc. 362 (2010), 2541-2558.

[8] P. Etingof, T. Schedler and A. Soloviev, Set-theoretical solutions to the quantum YangBaxter equation, Duke Math. J. 100 (1999), 169-209.

[9] Tatiana Gateva-Ivanova, Set-theoretic solutions of the Yang-Baxter equation, Braces, and Symmetric groups, arXiv:1507.02602 [math.QA].

[10] T. Gateva-Ivanova and M. Van den Bergh, Semigroups of I-type, J. Algebra 206 (1998), $97-112$. 
[11] Peter Hubert, Nearrings and a Construction of Triply Factorized Groups, doctoral dissertation, Johannes Gutenberg-Universitat in Mainz, 2005.

[12] Eric Jespers and Jan Okninski Binomial semigroups, J. Algebra 202 (1998), 250-275.

[13] D.S. Passman, The Algebraic Structure of Group Rings, Wiley, New York, 1977.

[14] D. J. S. Robinson, A Course in the Theory of Groups, Springer-Verlag GTM Vol. 80 (1982).

[15] Wolfgang Rump, Braces, radical rings, and the quantum Yang-Baxter equation, Journal of Algebra Volume 307, Issue 1, 1 January 2007, Pages 153-170.

[16] Ya. P. Sysak, Products of groups and local nearrings, Note. Mat. 28 (2008), suppl. n. 2. 177-214.

[17] M. Takeuchi, Survey on mached pairs of groups. An elementary approach to the ESS-LYZ theory, Banach Center Publ. 61 (2003), 305-331.

Ferran Cedó, Departament de Matemàtiques, Universitat Autònoma de Barcelona, 08193 Bellaterra (Barcelona), Spain

Tatiana Gateva-Ivanova, American University in Bulgaria, 2700 Blagoevgrad, and Institute of Mathematics and Informatics, Bulgarian Academy of Sciences, 1113 Sofia, Bulgaria

Agata Smoktunowicz, School of Mathematics, The University of Edinburgh, James Clerk Maxwell Building, The Kings Buildings, Mayfield Road EH9 3JZ, Edinburgh

E-mail: cedo@mat.uab.cat, Tatyana@aubg.edu, A.Smoktunowicz@ed.ac.uk 